\title{
EFFECT OF WALL PROXIMITY ON CROSS FLOW TIP LEAKAGE IN DIFFUSER AUGMENTED WIND TURBINES (DAWTs)
}

\author{
A.S. DAR ${ }^{1,2}$, Z.M. SHEIKH ${ }^{1,3}$ \& S.H.W. GILANI ${ }^{1}$ \\ ${ }^{1}$ Department of Mechanical Engineering, University of Engineering \& Technology, Lahore, 54890, Pakistan. \\ ${ }^{2}$ Department of Mechanical Engineering, Politecnico di Milano, 20156, Italy. \\ ${ }^{3}$ UM-SJTU Joint Institute, Shanghai JiaoTong University, Shanghai, 200240, China.
}

\begin{abstract}
Diffuser Augmented Wind Turbines (DAWTs) have been a highlight of research in small size wind turbines due to their potential capability of exceeding Betz Limit. Bringing a wall in close proximity of blade tip may have strong influences on physics of tip leakage flow. In current study, the effect of the diffuser wall on tip leakage flow is examined. Different tip gap to diffuser radius ratios are numerically studied in a two dimensional domain. As tip leakage flow in DAWTs has not been studied before, the methodology for the current work is adopted from similar research in gas turbines. Obtained results indicate that as the tip gap to diffuser radius ratio is reduced from 0.067 to 0.02 , tip leakage mass flow rate reduces to $6 \%$ and the total pressure loss increases by $56 \%$. This behavior of tip leakage flow is further elaborated by discussing the flow physics in the gap region.
\end{abstract}

Keywords: CFD, DAWT, flow separation, tip leakage.

\section{NOMENCLATURE}

$G$ : Tip gap to diffuser radius ratio.

$m$ : Tip leakage mass flow rate $(\mathrm{kg} / \mathrm{s})$.

$P$ : Total pressure loss $(\mathrm{Pa})$.

A: Tip speed ratio.

$U$ : Air stream velocity.

$V_{w}$ : Diffuser wall velocity.

\section{INTRODUCTION}

The conventional energy technologies have been indicated as prime contributors to the global warming and are proved to have hazardous environmental impacts. To add to this, the Earth today has potential reserves of fossil fuels up till 2112; with coal being the only fossil fuel left after 2042 [1]. This restates the energy issue for us as: 'To look for environmental friendly, sustainable and stable energy sources'. Of all the renewables, wind energy technology has become the most imminent one because of its vast growth and potential to solve the energy crisis on a long-term basis. Ackerman [2] reviewed wind energy characteristics and concluded it to be an excellent energy source for electricity production. Horizontal axis wind turbines (HAWTs) have been the most widely used large scale wind turbines for electricity generation on commercial level [3].

A Diffuser Augmented Wind Turbine (DAWT) is a modern type of wind turbine focused on increasing mass flow rate and thus attempting to exceed Betz Limit. It is necessarily a duct or nozzle, which makes use of the Venturi effect to produce a decrease in pressure; this decrease in pressure increases the velocity inside the diffuser and thus mass flow rate increases.

The history of DAWT dates back to 1920s when Alexander Betz theoretically studied and identified the potential of DAWT. However, he rendered the concept to be economically 
unfeasible for the time being [4]. DAWT again became a topic of research in 1950s and scientists started to perform theoretical and experimental studies on augmentation of mass flow rate by using a diffuser [5-7]. The DAWTs instigated a new surge of interest in 1970s when different scientists and companies including Igra [8, 9] and Gilbert \& Foreman [10] worked on the concept. The focus of these studies was to optimize the performance of DAWT, specifically targeting boundary layer separation. Foreman [11] also worked on the economic aspects of DAWT.

Only a small number of full-scale DAWT turbines have been built including Vortec-7, which is based on the work by Foreman [12]. Phillips [13] identifies advantages of DAWT in comparison with bare wind turbines, including benefits like low cut-in speeds, reduction in noise and turbulence intensity and lower axial loads.

In an attempt to prove the potential capability of DAWT to exceed Betz Limit, Hansen [14] performed a simple 1D analysis to conclude that Betz Limit can be exceeded, whereas, Werle and Presz [15] predicted that power augmentation is proportional to Betz curve.

Current researches on DAWT focus on understanding the physics of the flow through the diffuser [16] or defining different geometries for the diffuser. Ten Hoopen [17] studied flow behavior in DAWT, whereas, different types of geometries including flanged, conical and airfoil shaped cross-section have been developed and studied [3, 14, 18, 19].

Tip leakage is the flow through the gap between the tip of blade and casing wall; it is generated because of the pressure difference between the two sides of the blade (called pressure and suction sides). In gas turbines, tip leakage flow is responsible for $30 \%$ of the turbine losses [20]. The studies concerning tip leakage in gas turbine mainly concentrate on either the control of tip leakage flow or thermal effects of flow on blade tip. Gas turbine community has used various strategies to reduce tip leakage flow such as reducing discharge coefficient, modification of exit conditions and varying the aerodynamics of the blade [21-23]. Whereas, the heat transfer effects through tip leakage have also been studied to ensure minimum thermal stresses on blade tip [24, 25].

Computational fluid dynamics has been a well-documented technique to study tip leakage in gas turbines [20,26]. Use of numerical simulations for study of tip leakage flow dates back to early 90s when Liu studied the flow in tip gap using numerical simulations [27]. Since then various $2 \mathrm{D}$ and 3D models have been developed to study flow through gap [25, 28, 29].

Motivation behind the current research is to investigate tip leakage flow in DAWTs by employing techniques used by gas turbine community to study the effect of wall proximity on tip leakage flow. Tip leakage flow in DAWTs has rarely been studied; therefore, current research focuses on understanding the physics that governs tip gap fluid dynamics in DAWTs. In coming sections, details related to numerical simulations and obtained results are discussed.

\section{METHODOLOGY}

As mentioned earlier, methodology of studying tip leakage flow is adopted from similar studies in gas turbines. Primarily, tip leakage in gas turbines is modelled as a two-dimensional flow. Figure 1 shows a quasi-three-dimensional tip gap model developed to simulate flow through tip gap in DAWT. The narrow constant area channel simulates tip gap while pressure and suction sides of the blade have been modelled as inlet and outlet, respectively.

ANSYS Fluent is used as the solver to simulate the computational domain. In order to account for turbulence effects, a two equation turbulence model $(k-\omega \mathrm{SST})$ is used. Boundary conditions used are discussed in the following. 


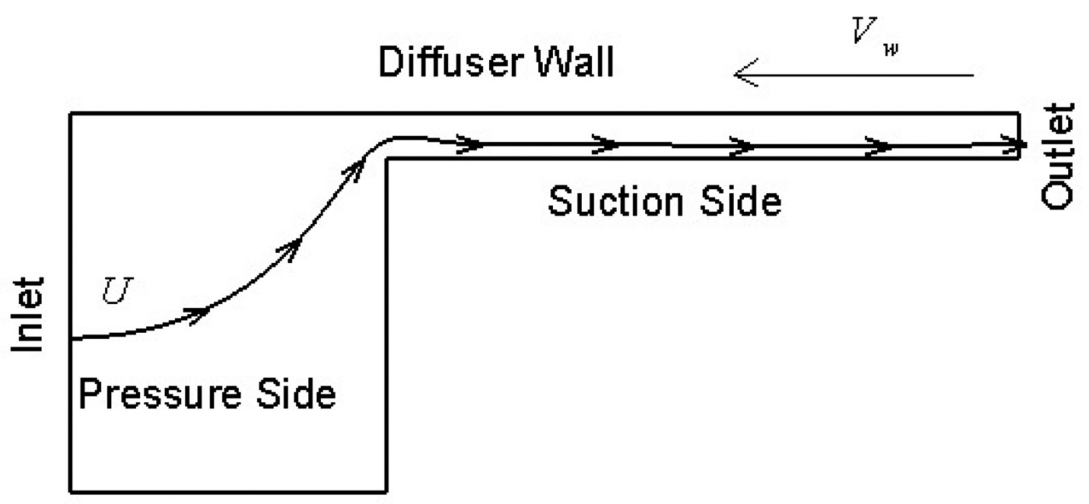

Figure 1: Model for studying tip leakage flow in DAWTs.

Inlet: Inlet is specified as a 'Pressure Inlet' boundary condition with a total pressure value of $45 \mathrm{~Pa}$.

Outlet: The outlet is specified as 'Pressure Outlet' with a pressure value of $0 \mathrm{~Pa}$. Pressure values for inlet and outlet boundary conditions are selected to approximate tip leakage flow conditions in wind turbines.

Diffuser Wall: The upper horizontal wall represents the diffuser surface and to simulate the relative motion between the blade tip and the diffuser wall, 'moving wall' boundary condition is used for diffuser wall. The velocity of moving wall is calculated by using ideal tip speed ratio formula [30]. The assumed radius of turbine is $1.5 \mathrm{~m}$ and number of blades are three. The moving wall velocity $V_{w}$ is assigned the opposite sign of tip speed velocity to simulate the movement of tip past the diffuser surface.

Wall: Rest of the boundary walls, i.e. the lower horizontal boundary, the corner boundary and lower boundary of tip gap channel are all assigned 'Wall' boundary conditions.

It should be noted that motivation behind the current study is to understand the effect of diffuser wall on tip leakage flow, and therefore, absolute values of boundary conditions are not important as far as they are kept consistent for every gap studied.

\section{RESULTS \& DISCUSSION}

In a bare turbine, the casing wall can be assumed to be at an infinite distance from the turbine; as this wall is brought close to the blade, it starts interacting with the flow through turbine. The tip gap has been non-dimensionalized by the diffuser radius to get non-dimensional tip gap to diffuser radius ratio $\mathrm{G}$. It is meaningful to study the effect of different tip gaps on the total pressure loss and tip leakage mass flow rate through the clearance. Therefore, eight values of $\mathrm{G}$ between 0.067 and 0.02 are studied. To ensure mesh independence, six meshes for each $\mathrm{G}$ are solved. Figure 2 shows mass flow rate and total pressure loss for $\mathrm{G}=0.033$ and 0.053 against mesh density. The results indicate that mesh of more than 3 million cells is enough to resolve the flow.

\subsection{Flow physics}

Tip leakage flow is complex in nature, involving interaction of multiple factors, such as tip clearance, pressure difference, blade velocity, etc. Based on the results, we put forward the 

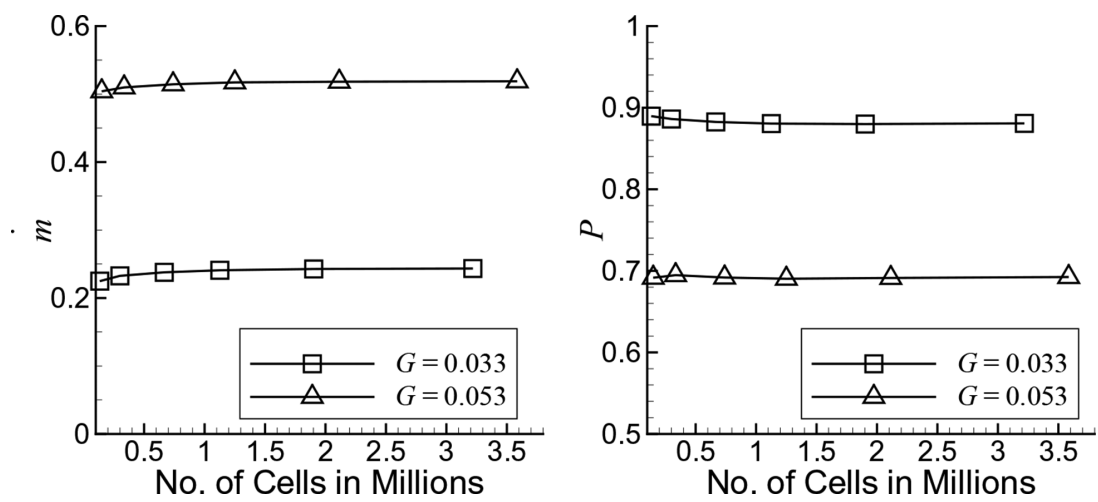

Figure 2: Mesh independence study (a) mass flow rate vs. number of cells (b) total pressure loss vs. number of cells.

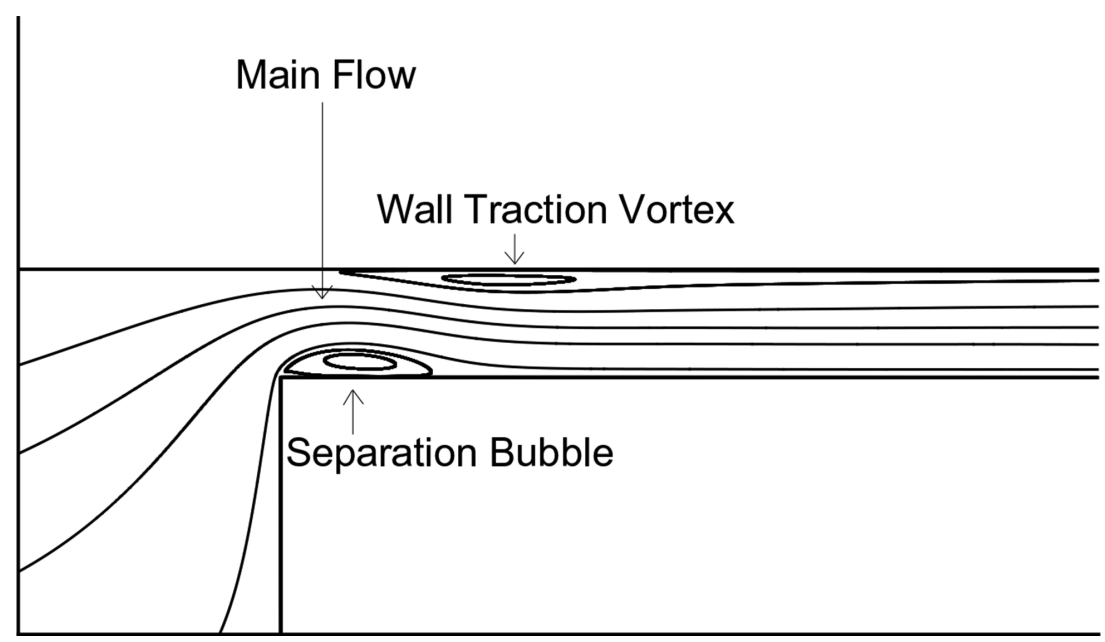

Figure 3: Schematic of flow through tip gap.

following explanation of the governing physics. As illustrated by Fig. 3, incoming flow from inlet creates a separation vortex at the tip. Moreover, backward moving diffuser wall generates a region of recirculating flow called wall traction vortex. Size and position of both separation and wall traction vortices are a strong function of tip gap. For instance, at large tip gap ratios, space for separation vortex to grow larger is available; therefore, its size is large. For larger tip gaps, shearing produced by diffuser wall motion is not very effective; therefore, mass flow rate through gap is large which pushes the wall traction vortex downstream. As diffuser wall is brought close to the blade tip, i.e. $G$ becomes small, separation vortex shrinks; however, shearing caused by moving diffuser wall becomes effective in mobilizing the flow near it. Stronger shearing leads to a stronger wall traction vortex, which then blocks the main flow and reduces the mass flow rate through tip gap. In the following section, results obtained from simulation are presented. 

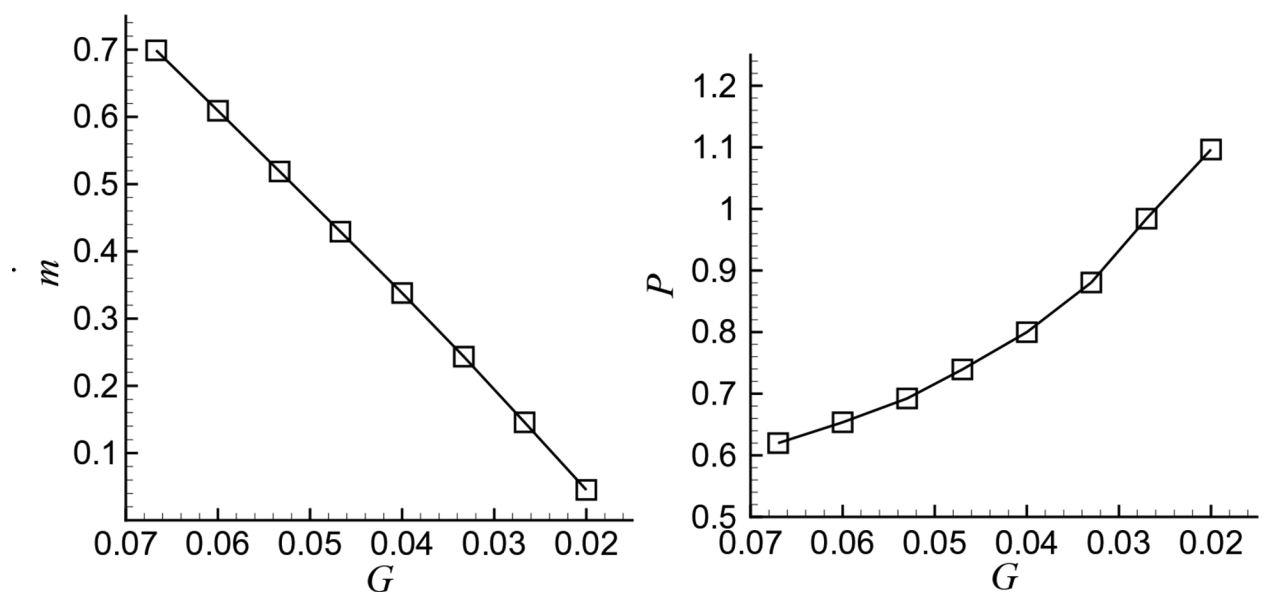

Figure 4: (a) Mass flow rate against $G$ (b) Total pressure loss against $G$.

\subsection{Results}

Variation of tip gap mass flow rate and pressure loss for different gaps is shown in Fig. 4. For largest value of $G$, mass flow rate of the tip leakage flow is largest. As tip gap $G$ is decreased, tip leakage mass flow rate decreases linearly. For the lowest value of $G$, the mass flow rate reduces to $6 \%$ of the mass flow rate at $G=0.067$. This significant decrease in mass flow rate suggests that in order to avoid leakage of useful momentum through tip gap, clearance between blade tip and diffuser wall should be minimized. Another important factor is the loss in total pressure across the clearance. As can be seen from Fig. 4, total pressure loss increases with decreasing $G$. For smallest $G$, total pressure loss is nearly 1.56 times the pressure loss for the largest $G$. In the following, effect of wall proximity on pressure and flow structure is discussed.

The separation vortex formed at the tip due to flow separation and wall-traction vortex generated by the opposite motion of casing wall interact with each other to govern the flow through the gap. Both vortices block the forward passage of the main flow and the flow propagates through the throat generated in between the two vortices.

From obtained numerical results shown in Fig. 5, it is observed that the separation bubble increases in size with the increase in $G$. The small size of separation bubble in lowest $G$ value is attributed to the fact that due to the relatively small space available, the vortex does not get enough space to grow. Whereas, it can be seen from Fig. 5 that wall traction vortex grows weaker and is pushed downstream by the main flow as $G$ is increased. It is evident from Fig. 5 that due to flow passage being blocked by separation and wall traction vortex, mass flow rate decreases monotonously with decreasing tip gap.

The pressure distribution on pressure side wall of the model is also shown in Fig. 5. As it can be seen from the figure, that decrease in tip gap ratio $G$ increases the pressure magnitude on pressure side of the blade. A higher magnitude of pressure on pressure side indicates more power production. Therefore, it is reasonable to conclude that a DAWT should lead to a higher power generation as compared to a bare turbine. 

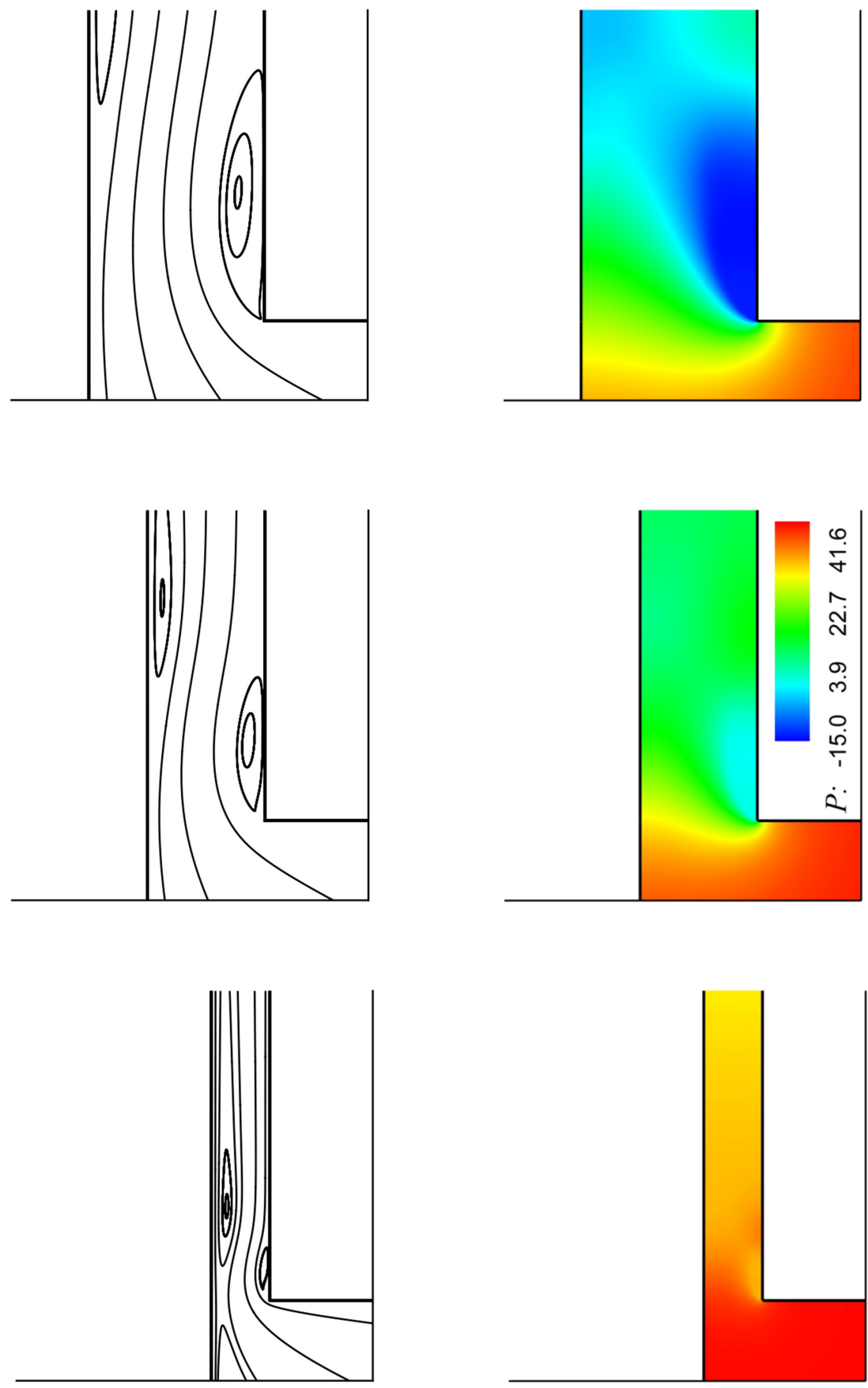

Figure 5: Flow structures and tip pressure distributions for various $\mathrm{G}$ values. 


\section{CONCLUSIONS}

The focus of this study is to investigate the effects of bringing a wall (casing) near a bare wind turbine. From the numerical study conducted, following conclusion can be drawn.

1. Flow through tip gap channel is governed by two major vortices: separation bubble and wall-traction vortex. The gap between the two vortices provides a passage for the main flow to propagate in forward direction. It is observed that separation vortex decreases in size while wall-traction vortex increases by bringing the wall closer to blade.

2. The effect of bringing wall closer to blade decrease the tip leakage mass flow rate but increases the pressure loss across the tip gap region.

3. Due to tip leakage, the pressure distribution on pressure side of blade or tip is also affected. Presence of diffuser wall reduces the tip leakage flow, and therefore, magnitude of pressure on pressure side of the blade increases which then should lead to a higher power output of wind turbine.

4. Reduction in tip gap ratio causes mass flow rate to drop significantly. Ideally, zero gap between blade tip and diffuser wall is desirable.

It is worth noting that the tip gap flow involves complex interaction between multiple factors and therefore requires further investigation in broad range of parameters.

\section{REFERENCES}

[1] Shafiee, S. \& Topal, E., When will fossil fuel reserves be diminished? Energy Policy, 37(1), pp. 181-189, 2009.

http://dx.doi.org/10.1016/j.enpol.2008.08.016

[2] Ackermann, T. \& Sder, L., An overview of wind energy-status 2002. Renewable and Sustainable Energy Reviews, 6(1-2), pp. 67-127, 2002.

http://dx.doi.org/10.1016/S1364-0321(02)00008-4

[3] Hjort, S. \& Larsen, H., A multi-element diffuser augmented wind turbine. Energies, 7(5), pp. 3256-3281, 2014.

http://dx.doi.org/10.3390/en7053256

[4] Betz, A., Energieumsetzungen in Venturidsen. Naturwissenschaften, 17(10), pp. 160 164, 1929. http://dx.doi.org/10.1007/BF01506436

[5] Sanuki, M., Studies on biplane wind vanes, ventilator tubes and cup anemometers (I). Papers in Meteorology and Geophysics, 1(1), pp. 81-132, 1950. http://dx.doi.org/10.2467/mripapers1950.1.1_81

[6] Iwasaki, M., The experimental and theoretical investigation of windmills. Reports of Research Institute for Applied Mechanics, 2(8), pp. 181-229, 1953.

[7] Sanuki, M., Kimura, S. \& Tsuda, N., Studies on biplane wind vanes, ventilator tubes and cup anemometers. Papers in Meteorology and Geophysics, 2(3-4), pp. 317-333, 1951. http://dx.doi.org/10.2467/mripapers1950.2.3-4_317

[8] Igra, O., Compact shrouds for wind turbines. Energy Conversion, 16(4), pp. 149-157, 1977. http://dx.doi.org/10.1016/0013-7480(77)90022-5

[9] Igra, O., Research and development for shrouded wind turbines. Energy Conversion and Management, 21(1), pp. 13-48, 1981.

http://dx.doi.org/10.1016/0196-8904(81)90005-4 
[10] Gilbert, B., Oman, R. \& Foreman, K., Fluid dynamics of diffuser-augmented wind turbines. Journal of Energy, 2(6), pp. 368-374, 1978.

http://dx.doi.org/10.2514/3.47988

[11] Foreman, K., Preliminary design and economic investigations of Diffuser-Augmented Wind Turbines (DAWT). 1981, Grumman Aerospace Corp., Beth-page, NY (USA). Research Dept.

[12] Phillips, D., Flay, R. \& Nash, T., Aerodynamic analysis and monitoring of the Vortec 7 diffuser-augmented wind turbine, 1999.

[13] Phillips, D.G., An investigation on diffuser augmented wind turbine design, 2003, ResearchSpace@Auckland.

[14] Hansen, M.O.L., Srensen, N.N. \& Flay, R., Effect of placing a diffuser around a wind turbine. Wind Energy, 3(4), pp. 207-213, 2000.

http://dx.doi.org/10.1002/we.37

[15] Werle, M.J. \& Presz, W.M., Ducted wind/water turbines and propellers revisited. Journal of Propulsion and Power, 24(5), pp. 1146-1150, 2008.

http://dx.doi.org/10.2514/1.37134

[16] Phillips, D., Richards, P. \& Flay, R., Diffuser development for a diffuser augmented wind turbine using computational fluid dynamics. Department of Mechanical, Engineering, the University of Auckland, New Zealand, 2008.

[17] Ten Hoopen, P., An experimental and computational investigation of a diffuser augmented wind turbine. M. Sc. Thesis, 2009.

[18] Barbosa, D.L., Vaz, J.R., Figueiredo, S.W., Silva, M.D.O.E., Lins, E.F. \& Mesquita, A.L., An investigation of a mathematical model for the internal velocity profile of conical diffusers applied to DAWTs. Anais da Academia Brasileira de Cimcias, 87(2), 2015.

[19] Isensee, G.M. \& Abdul-Razzak, H., Modeling and analysis of diffuser augmented wind turbine. International Journal of Energy Science, 2012.

[20] Wheeler, A.P. \& Sandberg, R.D., Direct numerical simulations of a transonic tip flow with free-stream disturbances. In ASME 2013 Turbine Blade Tip Symposium, American Society of Mechanical Engineers, 2013.

[21] Booth, T., Importance of tip leakage flow in turbomachinery design, tip clearance effects in axial turbomachinery. VKI Lecture Series, 5, 1985. http://dx.doi.org/10.1115/TBTS2013-2037

[22] Yamamoto, A., Tomlnaga, J., Matsunma, T. \& Outa, E., Detailed measurements of three-dimensional flows and losses inside an axial flow turbine rotor. In ASME 1994 International Gas Turbine and Aeroengine Congress and Exposition, American Society of Mechanical Engineers, 1994.

[23] Patel, K., Research on a high work axial gas generator turbine. SAE Technical Paper, 1980.

[24] Ameri, A.A., Steinthorsson, E. \& Rigby, D.L., Effects of tip clearance and casing recess on heat transfer and stage efficiency in axial turbines. In ASME 1998 International Gas Turbine and Aeroengine Congress and Exhibition, American Society of Mechanical Engineers, 1998.

[25] Wheeler, A.P., Atkins, N.R. \& He, L., Turbine blade tip heat transfer in low speed and high speed flows. Journal of Turbomachinery, 133(4), p. 041025, 2011.

http://dx.doi.org/10.1115/1.4002424

[26] Kavurmacioglu, L., Dey, D. \& Camci, C., Tip leakage flow simulation in AFTRF blade passages. von Karman Institute Lecture Series VKI-LS, 2, pp. 27-41, 2004. 
[27] Liu, J.-S. \& Bozzola, R., Three-dimensional Navier-Stokes analysis of tip clearance flow in linear turbine cascades. AIAA Journal, 31(11), pp. 2068-2074, 1993. http://dx.doi.org/10.2514/3.49123

[28] Camci, C. \& Shavalikul, A., Pressure side tip platform extensions for tip leakage control in axial turbines, 2008.

[29] Legras, G., Gourdain, N. \& Trebinjac, I., Numerical analysis of the tip leakage flow field in a transonic axial compressor with circumferential casing treatment. Journal of Thermal Science, 19(3), pp. 198-205, 2010. http://dx.doi.org/10.1007/s11630-010-0198-y

[30] Twidell, J. \& Weir, T., Renewable Energy Resources, Routledge: London and New York, 2015. 